\title{
Correcting errors in eye-position data arising from the distortion of magnetic fields by display devices
}

\author{
ROBERT T. CALDWELL and KEVIN S. BERBAUM \\ University of Iowa, Iowa City, Iowa \\ and \\ JOSH BORAH \\ Applied Science Laboratories, Bedford, Massachusetts
}

\begin{abstract}
Eye-position recordings have been used to produce detailed information about sampling and search behavior. The melding of magnetic head-tracking technology with eye-tracking systems has allowed for freer head movement, but not without problems. Although the requisite calibrations made before and after a reading might indicate good eye-tracking accuracy, point-of-gaze errors can occur if the reader leans toward a display, such as a film alternator. The error results from the characteristic that large metal masses can distort magnetic fields, leading to false magnetic sensor data. Corrections for this type of error have involved mapping the exact location and orientation of the magnetic sensor in the magnetic field by using a precision positioning device. We have devised a much cheaper method that relies on a fixture that holds the magnetic sensor in identical positions, with and without the distorting effect.
\end{abstract}

Accurate measurement of the eye positions of radiologists as they inspect radiographs has provided indirect evidence about radiologists' decisions (Nodine \& Kundel, 1987). Eye-position recordings have shown, for example, that most undetected pulmonary nodules have received prolonged visual attention (Kundel, Nodine, \& Krupinski, 1989, 1990). Krupinski (1996) has used eye-position recordings to document the experience levels of radiologists reading mammograms. Thus, knowing where radiologists look provides information about their search behavior and about radiologists themselves as "readers."

Although several eye-position recording methods have been developed over the years, most have involved compromises with regard to spatial accuracy and viewer constraint (Mulligan, 1997). Generally, the greater the degree of constraint on head position, the greater the possibility for more accurate measurement. Typical devices used to immobilize the head include bite bars, chin rests, and head rests. Unfortunately, constraining the head position can impede natural viewing behavior as well as lead to postural fatigue after periods of extended use.

Recently, eye-position recording systems in radiology perception research have been used in conjunction with

This work was supported by U.S. Public Health Service Grant RO1 CA 42453 from the National Cancer Institute, Bethesda, MD. Applied Science Laboratories retains a proprietary interest in the correction routine software that was added to the proprietary calibration routines, which precludes our sharing the source code of the routines. Correspondence concerning this article should be addressed to R.T. Caldwell, Department of Radiology, University of Iowa, Iowa City, IA, 52242 (e-mail: robt-caldwell@uiowa.edu). a head-mounted magnetic coil within a known magnetic field to measure head position (Berbaum et al., 1996; Krupinski, 1996; Krupinski, Nodine, \& Kundel, 1998). These measurements have been used with eye-position data to determine point of gaze. The major advantage of these systems is that they permit head movement without affecting the measurement of gaze.

Radiologists typically view X-rays on film alternators, which are motorized film storage units capable of holding several hundred radiographs. Unfortunately, the large metal mass often found in these film alternators can cause considerable distortion in the magnetic field. We have found that this distortion is particularly strong when the reader moves closer to the display. The distortion causes erroneous head-position data and consequently generates inconsistencies between where the radiologists are actually looking and what the eye-tracking system indicates as their point of gaze. In our laboratory, subjects are free to move their heads after calibration, but they are asked not to lean forward during the actual reading. Other researchers have used similar restrictions on viewing behavior in order to overcome the distortion problem (Krupinski, personal communication, 1996). Although these interventions have been effective in maintaining a more accurate recording of eye position, they limit the natural inspection behavior under study. Another approach, most often used in military applications of magnetic trackers, is to map and correct for the distorted magnetic field. This usually requires construction of an expensive, nonmetallic fixture that enables the magnetic sensor to be positioned at very precise and known positions and orientations. This method may be the only reasonable approach 


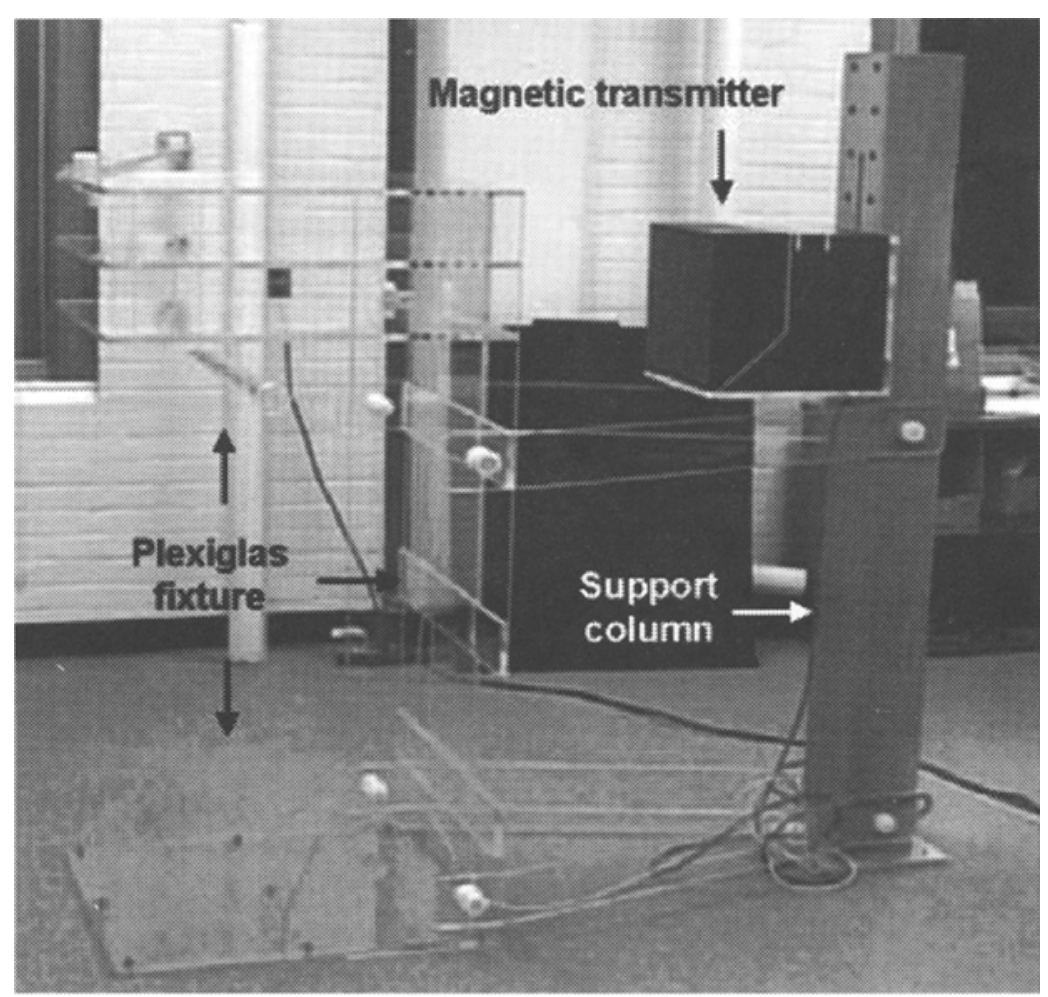

Figure 1. The nonmetallic positioning device was constructed of Plexiglas and was made immobile by attaching it to the magnetic support column, which itself was anchored to the concrete floor. A Plexiglas strap was fastened to the room wall in order to further stabilize the fixture. Nine pins were punched through the carpet, securing the Plexiglas base to the concrete floor.

for such applications, since the distorting metal (such as an aircraft or flight simulator) cannot easily be removed.

We have devised a variation of this solution that takes advantage of the fact that our distorting mass is on wheels. By measuring the spatial location and orientation of the sensor with and without the distortion, the magnitude of the distortion can be measured without the use of an expensive positioning device. The environment in which the magnetic tracker collects data is assumed to be benign and the data to be sufficiently accurate when the distorting metal has been removed. Corrections are computed by comparing magnetic tracker data in the benign state with data collected in the distorted environment, which is caused by the presence of the film alternator.

\section{MATERIALS AND METHODS}

Correction data were collected using an Applied Science Laboratories (ASL) Model $4100 \mathrm{H}$ head-mounted, eye-tracking system. This system includes a Big Bird magnetic head tracker (Ascension Technology Corporation, Burlington, VT) that consists of a magnetic field transmitter and magnetic field sensor. In actual use, the magnetic field sensor is attached to a head-mounted optical system and permits precise localization of head position within the magnetic field. The ASL eye-tracker software program EYEPOS/EYEDAT was used to examine preprocessed video data, eliminate arti- facts, identify the pupil and corneal reflection, and calculate point of gaze. The ASL program EYEHEAD was used to integrate eye and head data. Digital records were collected and stored in computer memory for each $1 / 60$ th sec of data sampled. These records included point of gaze on the image (output as $x, y$ coordinates) and viewing distance.

\section{Correction Apparatus and Procedure}

A nonmetallic fixture was constructed in order to permit repeated placement of the magnetic sensor in multiple positions in the observational space relative to the magnetic transmitter. Three $2 \mathrm{ft}^{2}$ planes of $1 / 2$-in. Plexiglas were stacked 5 in. apart. On each plane, a square grid was laid out at 5 -in. intervals, creating 25 equidistant intersects. Two threaded holes were drilled at each of the 75 positions, permitting the attachment and reattachment of the magnetic sensor at the same location. The entire Plexiglas jig was rigidly attached to the floor, the room wall, and to the immovable column that supported the magnetic transmitter (Figure 1).

A benign environment was created when the film alternator was moved far away from the transmitter. The spatial positions of the 75 locations were relative to the transmitter and were determined by using a mapping program developed by ASL. The program measured six parameters-the linear positions of the sensor relative to the transmitter along the $x-, y$-, and $z$-axes, and the angular orientations of the sensor to each of these axes - namely, the degree of azimuth, elevation, and roll. In the head-tracker transmitter axis system, the $x$-axis points out from the front of the transmitter toward the film alternator, the $y$-axis points out from the side of the transmitter, and the $z$-axis points down. If an observer were flying an airplane straight 


\section{A Mean Linear Error Near Film Alternator}

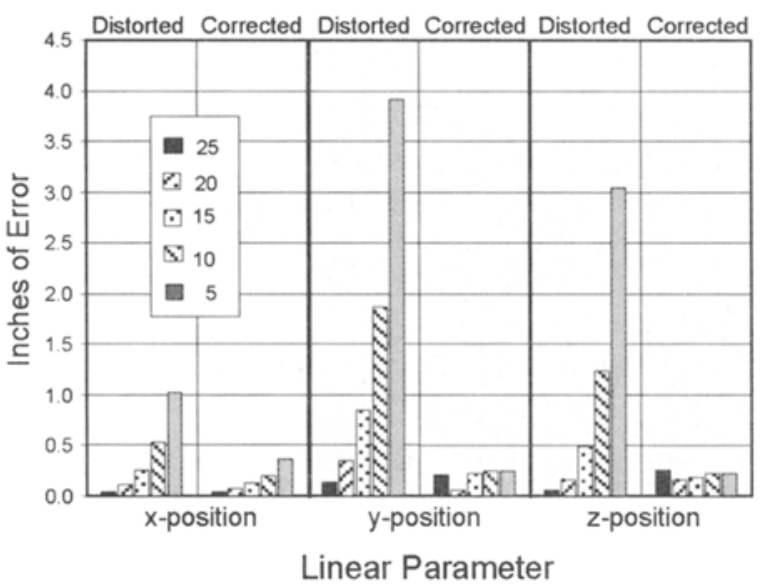

B Mean Angular Error Near Film Alternator

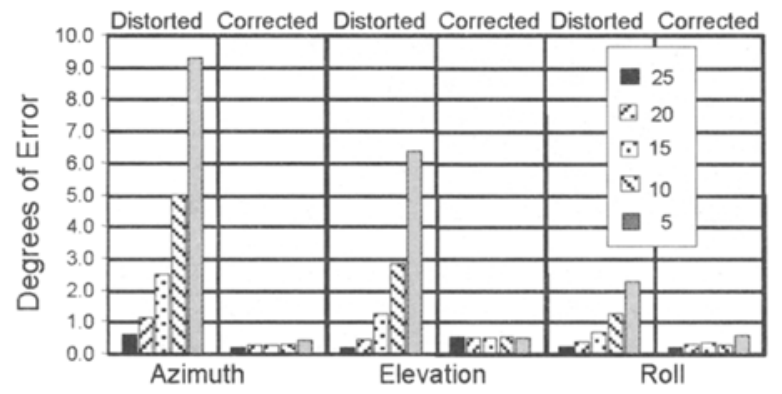

Angular Parameter

Figure 2. Side-by-side comparison of the errors between the distorted and benign environment and the corrected and benign environment for $(A)$ the three linear parameters and $(B)$ the three angular parameters, placed at distances ranging from 5 to 20 in. (box).

and level, the azimuth would be compass heading, elevation would be nose up/nose down orientation, and roll would be wing up/wing down orientation or bank angle.

After the positions and orientations of the sensor were measured at each of the 75 locations, the film alternator was moved back into place, creating a distorted environment. The sensor's positions and orientations were again measured, which, this time, indicated the magnitude of distortion caused by the metal mass in the film alternator. A polynomial equation (see the Appendix) developed by Ascension Technology Corporation used the coefficients from the mapping program for computing the corrected sensor data. The data gathering program corrected small orientation placement errors by simply subtracting orientation values that were measured under benign conditions from those measured under distorted conditions. A new version of ASL's E4000 EYEPOS program was written in order to read files containing the mapping polynomial coefficients and to use these to convert magnetic head tracking data before EYEHEAD integration computations were made.

The default coordinates of the sensor are parallel to the transmitter coordinates when the sensor cable extends away from the sensor in the same direction as the transmitter cable and when the sensor-mounting surface is parallel to the transmitter-mounting surface. The reference frame "attached" to the sensor can be rotated with respect to the sensor under software control. The EYEHEAD integration software rotates the sensor's coordinates, so that during calibration (with the subject's head still), the sensor coordinates will be parallel to the transmitter coordinates and, at the same head position, the orientation values will all be zero. The software treats each scene plane as being in the $y, z$ plane of a coordinate system so that, as the subject looks at the surface, the $y$-axis will extend to the subject's right, the $z$-axis will extend down, and the $x$-axis will extend out from the back of the surface (i.e., away from the subject). This means that if a surface is oriented in the vertical plane directly in front of the observer (and the transmitter), the coordinates for that surface will be more or less aligned with the transmitter coordinates.

\section{Efficacy of the Correction Method}

In order to determine the effectiveness of the correction method, the following procedure was performed three times, first using the corrected sensor data, then the noncorrected sensor data, and then again the corrected sensor data. Prior to each procedure, the eyetracking apparatus was calibrated to the observer's eye position, using a previously reported method (Berbaum et al., 1998; Berbaum et al., 1996).

\section{Procedure}

Four volunteers from the radiology department staff of the University of Iowa were asked to test the results of the correction-mapping program. Each observer sat in front of a nine-dot calibration grid positioned on a film alternator. The observers were instructed to move their heads freely while positioning each dot centrally within their field of view. As the observers stared at each dot, several seconds of fixation data were collected. The longest sustained fixation cluster within each dot-viewing interval was used to assess fixation accuracy. The distance from the point of fixation to the center of the calibration dot and each observer's viewing distance was used to compute the calibration error in terms of the observer's visual angle. Each observer's accuracy was indexed by the median, mean, and standard deviation of error distance and angle. Averages of these values were then computed. Data were collected at three viewing positions-one in which the observer sat approximately 28 in. from the display surface of the film alternator, one in which the observer moved to within $14 \mathrm{in}$. of the film alternator, and the last, in which the observer returned to the initial position.

\section{Results}

In order to demonstrate the magnitude of distortion and that of the correction, the 75 positions were sorted into five groups. Fifteen positions in each group defined a plane parallel to the display surface of the film alternator, the positions were placed in 5 -in. increments from the display surface. The difference in measurement between the benign and distorted and the benign and corrected environments determined the amount of error at each sensor location. Mean values were calculated for the distorted and corrected errors for each of the three linear positions, $x, y$, and $z$, and for the three angular orientations. A comparison of these means at 5 -in. increments relative to the film alternator is presented in Figure 2. The largest linear error was in the $y$ position, and the largest angular error was in the azimuth. If a radiologist were to lean forward 5 to $10 \mathrm{in}$. from the film alternator in order to carefully examine a radiograph, the head-position measurement could be off by as much as 1 in. in or out, 4 in. side to side, and 3 in. up and down. Similarly, the angular distortion could be off by $9^{\circ}$ azimuth, $6^{\circ}$ elevation, and $2^{\circ}$ roll. 


\section{Mapping Program Error Correction}
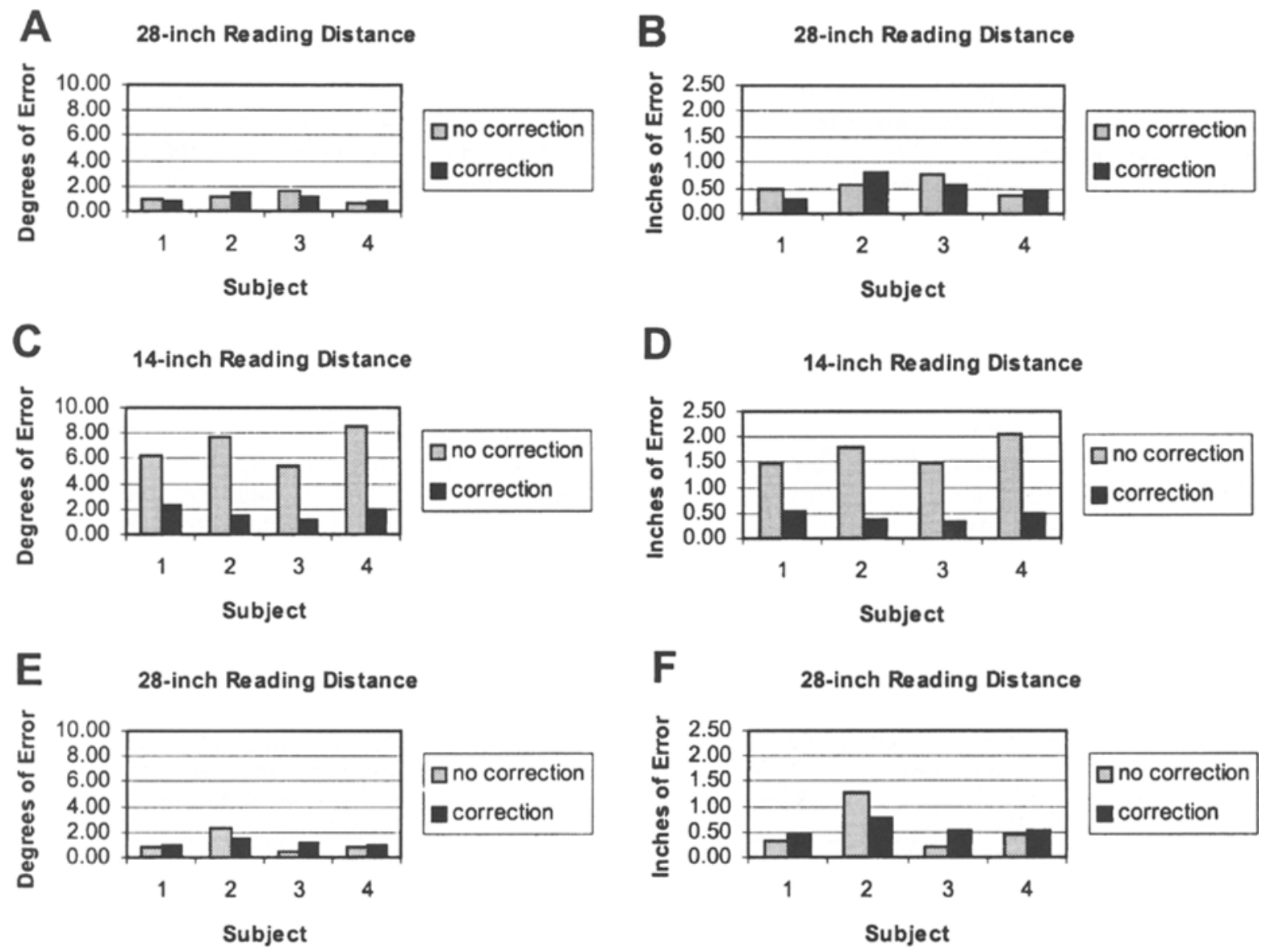

Figure 3. The magnitude of point-of-gaze correction in $(A, C, E)$ visual angles and in $(B, D, F)$ inches with the use of the mapping program that supplied corrected spatial coordinates during a dynamic reading of a nine-dot calibration grid. The eyetracking apparatus was first calibrated to each subject's eyes. Four subjects were allowed free head movement at viewing distances of 28-in., 14-in., and again 28-in. Using EYEHEAD software, measurements were computed (ASL) from the 3 linear and 3 angular parameters.

After correcting for linear and angular distortion, the errors between the corrected and benign data were nearly uniform and, in most cases, greatly reduced. The increased distortion caused by the proximity of the large metal mass was decreased.

Observer performance in corrected vs. noncorrected environments. Measures of error in the measurement gaze were analyzed using a repeated measures analysis of variance (ANOVA) with a within-subjects factor for field distortion correction versus no correction and another within-subjects factor for the nine locations in the visual field at which the measurements were collected (Figure 3).

Mapping and correcting for the magnetic field distortion substantially improved the accuracy of gaze measurement at the closer viewing distance (6.98 vs. 1.45 degrees of error for uncorrected and corrected measure- ment, respectively $[F(1,3)=44.23, p=.0069]$. There was also a statistically significant effect of location in the visual field at which the measurements were made $[F(8,24)=11.52, p=.0000]$ and a statistically significant interaction between correction and location $[F(8,24)$ $=6.19, p=.0002]$. These two effects reflect that the magnitude of error in gaze measurement generated by the magnetic field distortion and the amount of reduction in that error by the correction depended on where the observer fixated.

Similar results were produced using the same type of ANOVA when error was expressed in inches. Mapping and correcting for the magnetic field distortion substantially improved the accuracy of gaze measurement at the closer viewing distance ( $1.70 \mathrm{vs.} 0.42 \mathrm{in}$. of error for uncorrected and corrected measurement, respectively) $[F(1,3)=91.31$, $p=.0024]$. There was also a statistically significant effect 
of location in the visual field at which the measurement was made $[F(8,24)=13.63, p=.0000]$ and a statistically significant interaction between correction and location $[F(8,24)=4.39, p=.0022]$.

Error in calibration measures taken at the 28 -in. viewing distance, whether taken before or after measurements had been taken at the 14-in. viewing distance, showed no significant differences for corrected and noncorrected measurements. These measurements were comparable to those obtained for the corrected measurement at the 14in. viewing distance, with errors of 0.54 in. or $1.1^{\circ}$ before and 0.57 in. or $1.1^{\circ}$ after the 14 -in. measurements were made.

Viewing distance was not significantly different when the correction was withheld or applied in the far viewing before near viewing condition $(28.0$ vs. 28.3 in.) $[F(1,3)=$ $1.13, p=.3653]$, in the near viewing condition (14.1 vs. 14.2 in.) $[F(1,3)=0.53, p=.5202]$, or in the far viewing after near viewing condition (28.6 vs. 28.4$)[F(1,3)=0.26$, $p=.6426]$.

\section{DISCUSSION}

Experiments using $1-\mathrm{cm}$ simulated pulmonary nodules as targets have generally considered a point-of-gaze measurement to be good when it falls within a centimeter of the target or within approximately $1^{\circ}$ of visual angle at a 70-cm (28-in.) viewing distance (Carmody, Nodine, \& Kundel, 1981; Kundel, Nodine, \& Carmody, 1978; Nodine, Kundel, Toto, \& Krupinski, 1992). This accuracy of measurement is determined by before and after calibrations. Prior to each case, the reader was presented a nine-dot grid. The readers were directed to sit back, keep their heads still, and look at the nine dots, one at a time. As the readers' eyes moved from dot to dot, their points of gaze were tracked. When the point of gaze was within acceptable limits, a radiograph was rolled into place and the readers were free to move their heads. After the readers examined each case, they were again presented the nine-dot grid. The drift between the first and second grids was a measure of the accuracy of the eye-tracking system. Even when the before and after calibrations appeared to be good, the point-of-gaze measurement could still have been distorted during the intervening case reading. This became apparent when one reader moved closer to inspect an area of interest on the radiograph. As a feature was pointed to and discussed in detail, the eyeposition cursor tracked a few inches off.

Our data confirm that as one moves forward, toward the film alternator, the error in sensor location increases dramatically. Application of the correction polynomial can reduce this error. The largest error, and the most correctable, was found in viewing distances between 5 and $15 \mathrm{in}$. from the film alternator. This range is typical of the distance used by radiologists during close inspection of radiographs. In a few instances, the corrected data were slightly worse (e.g., with the roll and the $y$ and $z$ positions 25 in. from the film alternator). This occurred only with positions that were affected least by the distortion and was of nominal consequence compared with the robust error correction that occurred when the subjects moved forward, toward the film alternator.

It is difficult to determine analytically the effect that magnetic, head-tracking system errors have on the accuracy of gaze measurement, because taking this measurement is a three-dimensional problem that involves many variables. We first measured magnetic distortion in the static field surrounding the display. An error of a few inches, or degrees, in one parameter does not necessarily mean that the point-of-gaze measurement will be off by the same amount. No matter how large the error in the magnetic system data, the gaze measurements will remain accurate if the subject maintains the same head position as that during calibration. The eye-head integration software forces the orientation angles to be $0^{\circ}$ for the head position held during calibration. If position values are in error, the system will compute an erroneous spatial position for the eyeball, but will accurately construct a vector from that erroneous position to the proper gaze points on the calibration display surface.

When the subject moves after calibration, quantitative statements can be made with regard to error if some simplifying assumptions are also made. If the position data were accurate during calibration, and the only error caused by head movement was change in head azimuth angle, then the gaze vector reported by the system will have a direction that is in error by the same angle as that for the change in azimuth error from the original position. In terms of inches, on the viewing surface, the error depends on the current distance from the eye to the surface and the orientation of the surface. If gaze is perpendicular to the viewing surface, the gaze-angle error will be the distance from the eye to the surface, multiplied by the tangent of the error angle. However, if gaze intersects the surface at a highly skewed angle (i.e., very different from $90^{\circ}$ ), a small error in gaze angle will translate into a much larger distance on the surface.

To better demonstrate the effectiveness of our correction procedure, we measured point of gaze within a dynamic viewing field. In every instance, when the subjects moved closer to the display, significant error was measured. Very little error was measured when the subjects sat back at a distance commonly used for calibration. Thus, relying on calibration readings taken before and after a radiograph is read does not necessarily result in an accurate point-of-gaze measurement, especially if free head movement is allowed during actual data recording.

\section{Conclusion}

Our correction method allows one to avoid the expensive alternative often thought necessary for measuring location and orientation in a distorted field. The method described here results in more accurate data collection and extends the working range of the reader with minimum disruption of natural search behavior. It is very unlikely that any two laboratories will have identical types of distortions in their 
environments. We have shown that the film alternator in our laboratory distorts the magnetic field of the headtracking apparatus and interferes with accurate headposition measurements. We have also shown the degree to which this distortion can be eliminated by applying a correction polynomial. The general form of the correction polynomial is presented in the Appendix.

\section{REFERENCES}

Berbaum, K. S., Franken, E. A., JR., Dorfman, D. D., Miller, E. M., Caldwell, R. T., Kuehn, D. M., \& Berbaum, M. L. (1998). Role of faulty visual search in the satisfaction of search in chest radiography. Academic Radiology, 5, 9-19.

Berbaum, K. S., Franken, E. A., Jr., Dorfman, D. D., Miller, E. M., Krupinski, E. A., Kreinbring, K., Caldwell, R. T., \& Lu, C. H. (1996). Cause of satisfaction of search effects in contrast studies of the abdomen. Academic Radiology, 3, 815-826.

Carmody, D. P., Nodine, C. F., \& Kundel, H. L. (1981). Finding lung nodules with and without comparative visual scanning. Perception \& Psychophysics, 29, 594-598.

KRUPINSKI, E. A. (1996). Visual scanning patterns of radiologists searching mammograms. Academic Radiology, 3, 137-144.
Krupinski, E. A., Nodine, C. F., \& Kundel, H. L. (1998). Enhancing recognition of lesions in radiographic images using perceptual feedback. Optical Engineering, 37, 813-818.

Kundel, H. L., Nodine, C. F., \& Carmody, D. P. (1978). Visual scanning, pattern recognition and decision making in pulmonary nodule detection. Investigative Radiology, 13, 175-181.

Kundel, H. L., Nodine, C. F., \& Krupinski, E. A. (1989). Searching for lung nodules: Visual dwell indicates locations of false-positive and false-negative decisions. Investigative Radiology, 24, 472-478.

Kundel, H. L., Nodine, C. F., \& KRUPinsKi, E. A. (1990). Computerdisplayed eye position as a visual aid to pulmonary tumor interpretation. Investigative Radiology, 25, 890-896.

Mulligan, J. B. (1997). Image processing for improved eye-tracking accuracy. Behavior Research Methods, Instruments, \& Computers, 29, 54-65.

Nodine, C. F., \& KUNDEL, H. L. (1987). Using eye movements to study visual search and to improve tumor detection. RadioGraphics, 7 , 1241-1250.

Nodine, C. F., Kundel, H. L., Toto, L. C., \& Krupinski, E. A. (1992). Recording and analyzing eye-position data using a microcomputer workstation. Behavior Research Methods, Instruments, \& Computers, 24, 475-485.

SLABAUGH, G. G. (1999). Computing Euler angles from a rotation matrix. Retrieved on August 6, 2000 from http://users.ece.gatech.edu/ $\sim$ slabaugh/personal/research/euler/euler.pdf.

\section{APPENDIX}

Note-Polynomial derived by Ascension Technology Corporation, Burlington, VT.

For $x, y$, and $z$, the correction equations take the general form

$$
M_{\mathrm{c}}=M_{\mathrm{m}}+\sum A_{\mathrm{n}} * x_{\mathrm{m}}^{\mathrm{Pxn}} * y_{\mathrm{m}}^{\mathrm{Pyn}} * z_{\mathrm{m}}^{\mathrm{Pzn}},
$$

where $M_{\mathrm{c}}$ is the corrected measurement for $x, y$, or $z ; M_{\mathrm{m}}$ is the corresponding measured value of $x, y$, or $z$ added to a polynomial. The summation operator is used to describe a polynomial with each term (n) consisting of a constant coefficient $A_{\mathrm{n}}$ multiplying the three measured position values $x_{\mathrm{m}}, y_{\mathrm{m}}$, and $z_{\mathrm{m}}$, raised to various powers Pxn, Pyn, and Pzn.

For the Euler angles azimuth, elevation, and roll, the procedure is a bit more complicated because Euler angles cannot simply be added together. First a correction value is computed using equations in the form

$$
M_{\mathrm{c}}=\sum A_{\mathrm{n}} * x_{\mathrm{m}}^{\mathrm{Pxn}} * y_{\mathrm{m}}^{\mathrm{Pyn}} * z_{\mathrm{m}}^{\mathrm{Pzn}},
$$

where $M_{\mathrm{c}}$ is a correction value for azimuth, elevation, or roll. Note that measured azimuth, elevation, and roll values do not appear in the correction polynomial. Rotation matrices are then computed from both the measured Euler angles and the computed correction values (see above). Rotation matrices take the form

$$
\begin{aligned}
& \mathrm{RM}= \\
& \begin{array}{|lll}
\cos E * \cos A & \cos E * \sin A & -\sin E \\
-\cos R * \sin A & \cos R * \cos A & \sin R * \cos E \\
+\sin R * \sin E * \cos A & +\sin R * \sin E * \sin A & \\
\sin R * \sin A & \sin R * \cos A & \cos R * \cos E \\
+\cos R * \sin E * \cos A & +\cos R * \sin E * \sin A &
\end{array}
\end{aligned}
$$

where $A, E$, and $R$ are azimuth ${ }_{\mathrm{c}}$, elevation ${ }_{\mathrm{c}}$, and roll $\mathrm{c}_{\mathrm{c}}$, respectively. A correction matrix (CM) is then formed by the matrix multiplication

$$
\mathrm{CM}=\mathrm{SM} * \mathrm{DM},
$$

where SM is the rotation matrix formed from the measured azimuth, elevation, and roll values, and DM is the rotation matrix formed from the azimuth, elevation, and roll correction values.

The nine elements of this $\mathrm{CM}$ are defined generically by

$\left|\begin{array}{lll}\mathrm{CM}(1,1) & \mathrm{CM}(1,2) & \mathrm{CM}(1,3) \\ \mathrm{CM}(2,1) & \mathrm{CM}(2,2) & \mathrm{CM}(2,3) \\ \mathrm{CM}(3,1) & \mathrm{CM}(3,2) & \mathrm{CM}(3,3)\end{array}\right|$.




\section{APPENDIX (Continued)}

Finally, the corrected azimuth, elevation, and roll angles can be extracted from the matrix above by using the equations

$$
\begin{aligned}
& \text { elevation }=-\arcsin [\mathrm{CM}(1,3)], \\
& \text { azimuth }=\arctan [\mathrm{CM}(1,2) / \mathrm{CM}(1,1)],
\end{aligned}
$$

and

$$
\text { roll }=\arctan [\mathrm{CM}(2,3) / \mathrm{CM}(3,3)] .
$$

For a thorough discussion on Euler angles and rotation matrices, see Slabaugh (1999).

(Manuscript received June 10, 1999;

revision accepted for publication August 24, 2000.) 